\title{
La muerte súbita aumenta durante ejercicios vigorosos pero el entrenamiento físico la disminuye
}

Triggering of Sudden Death from Cardiac Causes by Vigorous Exertion. Albert C, Mittleman M, Chae C, et al. N.Engl. J.Med.2000;343:135561

\section{Objetivo}

Evaluar el rol de la actividad física en la aparición o prevención de la muerte súbita.

Diseño

Caso cruzado anidado del Physicians Health Study.

\section{Lugar}

Massachusetts, EEUU

\section{Pacientes}

Se realizó el seguimiento a 21.481 médicos hombres durante 12 años, cuya edad oscilaba entre 40 a 84 años y no tenían historia previa de enfermedad cardiovascular.

\section{Evaluación de factores pronósticos}

Al comenzar el estudio los médicos completaron un cuestionario sobre sus factores de riesgo cardiovascular, tipo de alimentación y frecuencia con que realizaban ejercicios físicos vigorosos.

\section{Medición del resultado principal}

Se registraron los casos de muerte súbita ocurridos durante el período de exposición al ejercicio físico vigoroso, definido como 30 minutos de ejercicio y 30 minutos después del ejercicio. Se calculó el riesgo de muerte súbita durante la actividad física vigorosa comparado con muerte súbita sin relación con actividad física.Se midió la incidencia de muerte súbita en relación al número de episodios u horas de actividad física durante el seguimiento.

\section{Resultados principales}

Durante los 12 años de seguimiento, se registraron 122 casos de muerte súbita entre los 21.481 participantes del estudio. Las carac- terísticas basales mostraban que los casos tenían significativamente más edad, historia de tabaquismo, diabetes e hipertensión, y menor consumo de alcohol y pescado. Veintitrés muertes súbitas estuvieron relacionadas con la actividad física vigorosa. El riesgo relativo de muerte súbita durante el período de exposición al ejercicio físico vigoroso fue de 16.9 (IC 95\% 10.5-27). Sin embargo el riesgo absoluto de muerte súbita durante cualquier episodio particular de ejercicio físico vigoroso fue extremadamente bajo (1 muerte súbita por 1.42 millones de episodios de ejercicio físico vigoroso o personas-horas), comparado con 1 muerte súbita por cada 23 millones de personas horas. El exceso de riesgo absoluto* es de 1 muerte súbita por cada 1.51 millones de episodios de ejercicios vigorosos. Efecto de la actividad física habitual sobre el riesgo de muerte súbita asociada al esfuerzo.

\begin{tabular}{l|l|l|l}
$\begin{array}{l}\text { Frecuencia habitual de actividad } \\
\text { Fisica vigorosa }\end{array}$ & $\begin{array}{l}\text { Muerte } \\
\text { Súbita } \\
\text { Total }\end{array}$ & $\begin{array}{l}\text { Muerte } \\
\text { Súbita } \\
\text { Relacionada } \\
\text { con el Esfuerzo }\end{array}$ & $\begin{array}{l}\text { Riesgo relativo } \\
\text { IC } 95 \%\end{array}$ \\
\hline$<1$ vez por semana & 32 & 3 & $74.1(22-249)$ \\
\hline $1-4$ por semana & 67 & 13 & $18.9(10.2-35)$ \\
\hline$>5$ veces por semana & 23 & 7 & $10.9(4.5-26.2)$ \\
\hline
\end{tabular}

Para muerte súbita global aquellos que hacían menos de una actividad física vigorosa semanal comparado con los que hacían más de 5 por semana, tuvieron un riesgo de $1.36(0.76-2.43)$.

\section{Conclusiones}

La actividad física vigorosa aumenta el riesgo de muerte súbita, pero en términos absolutos el riesgo es muy bajo. Realizar ejercicio físico vigoroso de manera habitual disminuye el riesgo de muerte súbita asociado con la práctica de este. No se encontró en este estudio una disminución del riesgo de muerte súbita global en aquellos que realizaban actividad física vigorosa.

Fuente de financiamiento: Grants del Instituto Nacional de Salud (NIH), EEUU

\section{Comentario}

La muerte súbita de causa cardiovascular ocurre durante o inmediatamente después del ejercicio físico.Estas pueden ser en personas con cierto grado de entrenamiento en competencias oficiales, como también en individuos de todas las edades que realizan actividades recreacionales.

La enfermedad ar terioesclerótica de las arterias coronarias es la causa de la mayoría de las muertes relacionadas con la actividad física (especialmente en personas de mas de 35 años, esten entrenadas o no).De todas las muertes súbitas que ocurren, alrededor de un $10 \%$ pueden vincularse con actividad física vigorosa1. Los resultados de mas de 50 estudios epidemiológicos de tipo observacionales, han demostrado que la falta de actividad física y el estilo de vida sedentario son factores de riesgo para el desarrollo o la progresión de enfermedad coronaria, y la actividad física aeróbica de moderada intensidad los disminuye2.

Existe una paradoja claramente marcada en el presente estudio;el ejercicio físico vigoroso puede incrementar el riesgo de muerte súbita por causa cardiovascular y también ofrecer protección para este riesgo en aquellas personas previamente entrenadas.

El motivo por el cuál no se encontró en este estudio una disminución de la muerte súbita global en aquellos sujetos que realizaban más actividad física puede deberse a distintos motivos básicamente relacionados con la manera de medir actividad física.
Por un lado se clasificó el tipo de actividad física (vigorosa, moderada o leve) por cuestionarios que completaban los participantes y no por personas calificadas para ello, esto provoco falta de uniformidad en la clasificación y pudo haber cambios en los niveles de actividad física durante el seguimiento. Por otro lado el ejercicio regular de intensidad moderada no fue asignado específicamente por lo tanto las personas que lo practican habitualmente pudieron ser clasificados como sedentarios.Los estudios que sí midieron actividad física de manera más rigurosa mostraron una disminución del riesgo de muerte súbita global asociado con mayor actividad física ${ }^{3}$. Desde el punto de vista fisiopatológico el motivo de la muerte súbita por el ejercicio físico vigoroso, obedece básicamente a dos causas: 1 - Activación del sistema nervioso simpático que promueve la ruptura de placas ateromatosas en las arterias coronarias.

2 - Desencadenamiento de fibrilación ventricular en el tejido previamente infartado.

Ambos mecanismos son contrarrestados por el aumento del tono vagal que presentan las personas físicamente activas.

Entonces, los beneficios de la actividad física global superan largamente el extremadamente bajo riesgo de muerte súbita relacionada al esfuerzo.Por lo cuál los resultados de este estudio de ninguna manera deben afectar la recomendación de realizar actividad física regular cuyo beneficio sobre la salud está claramente demostrado.

Dr. Christian Rhaiel [ Unidad de Medicina Familiar y Preventiva del Hospital Italiano de Buenos Aires ]

*Ver glosario

Referencias

1) Siscoick DS, Weiss NS, Fletcher RH.The incidence of primary cardiac arrest during vigourous exercise.N Engl J Med 1984;311:874-7

2) Morris JN, Everitt MG, Pollac R.Vigorous exercise in leisure time :protection against CHD.Lancet 1980;2: 1207-10

3) Leon AS, Connet J, Jacobs DR,et al.Leisure time physical activity levels and risk of CHD and death:the Mulitple Risk Factor Intervention Trial. JAMA 1987;258:2388-95

${ }^{*}$ Caso cruzado anidado:Para cada sujeto que tuvo un evento (ej.Caso con muerte súbita), se registra si ocurrió durante un episodio de ejercicio vigoroso o actividad habitual.Se puede determinar entonces en una población que proviene de una cohorte (estudio anidado) el riesgo de muerte súbita en períodos de actividad física vigorosa y compararlos con el riesgo de muerte súbita en aquellos con menor actividad.

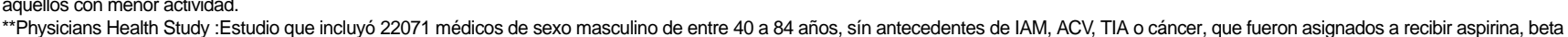
caroteno, o ambos, en un diseño randomizado, controlado con placebo, 2 × 2 factorial. 